\title{
Towards a systematic way to correct density functional approximations
}

\author{
Andreas Savin* \\ CNRS, UMR7616, Laboratoire de Chimie Théorique, F-75005 Paris, France. and \\ UPMC Univ Paris 06, UMR7616, Laboratoire de Chimie Théorique, F-75005 Paris, France.
}

(Dated: February 3, 2014)

\begin{abstract}
In order to simulate the exact universal density functional, approximations are nowadays constructed by permitting more flexibility in its ansatz. In view of the difficulty of defining a systematically improvable form for it, this paper argues that an alternative way could be considered. It falls within the class of hybrid functionals with multi-determinant wave functions. The parameter controlling the hybridization is considered as variable. The invariance of the exact result with respect to changes in this variable is used to introduce information about the system under consideration, and to correct the density functional result. The construction considered in this paper accelerates convergence from the model system to the physical one, in the vicinity of the latter. The method, at the present level of implementation, should be seen as a starting point for further development, and not necessarily as a computationally advantageous tool.
\end{abstract}

\section{INTRODUCTION}

The celebrated Hohenberg and Kohn paper [1] suggested that there was a systematic way of going beyond the local density approximation by using a gradient expansion. However, it turned out quickly that the first correction, the gradient expansion approximation, did not produce an improvement [2]. It took more than a decade to make progress, using generalized gradient expansions [3], and even more to arrive at hybrid methods [4]. Presently, the wide-spread strategy to improve density functionals is to use parameters (as a rule fitted on experimental data), and validate the newly produced functional by comparing the result with large sets of experimental reference data. The initial goal of a method providing a systematic improvement has thus been transformed into that of finding a more flexible ansatz that hopefully approaches the exact functional.

The aim of the present paper is less ambitious, in the sense that no approximation for the universal density functional is searched. However, it retains a density functional approximation as a starting point, and tries to improve on it systematically. Whether such an approach can lead to a practical method needs yet to be shown.

The approach considered in this paper consists in the following steps:

1. construct a model Hamiltonian, using a hybrid density functional approximation,

2. calculate the energy of this model system, and its first derivative with respect to a parameter defining the hybridization,

3. make an estimate of the correction to the density functional approximation using the first derivative.

This sequence can be continued by either using higher derivatives, or by considering new values of the parame-

*Electronic address: andreas.savin@lct.jussieu.fr ter. Thus, an improvement is achieved by adding more information of the specific system under study, that can be provided by the family produced by the variation of the parameter of the model.

The structure of the paper is the following. First, the method is described. Next, some simple numerical examples are given. Finally, other similar approaches are mentioned.

The central result of the paper is given by the equations 24 and 25.

\section{METHOD}

\section{Model Hamiltonian from density functional calculation}

In hybrid density functional calculations, the ground state energy is computed via:

$$
\mathcal{E}_{0}=\min _{\Psi}\left(\left\langle\Psi\left|T+V_{n e}+W(\mu)\right| \Psi\right\rangle+\overline{\mathcal{E}}_{h x c}\left[n_{\Psi}, \mu\right]\right)
$$

$T$ is the operator for the kinetic energy, $V_{n e}$ is the local one-particle operator for external potential (for the interaction between electrons and nuclei), and $W$ is a local two-particle operator. $\overline{\mathcal{E}}_{h x c}$ is an approximation to the universal Hartree-exchange-correlation functional, calculated from the density $n$. The subscript to $n$ indicates that is obtained from the $N$-electron wave function $\Psi$. Notice the presence of the parameter $\mu$. As $W$ depends on it, $\overline{\mathcal{E}}_{h x c}$ depends not only on $n$, but also on $\mu$.

In the Kohn-Sham method, $W=0$. In the present formalism, as one considers $W \neq 0$, the minimizing wave function $\Psi$ gets a multi-determinant character. For practical applications it will be important to use such forms of $W$ that do not introduce large errors when only a few determinants are used. For example, already a weak interaction can switch on the coupling between nearly degenerate states of the non-interacting system. In this paper, $\Psi$ has, for $W \neq 0$, multi-determinant character, although in literature the term "hybrid" is often used for 
the approximation in which $\Psi$ is described by a single Slater determinant.

The stationarity condition with normalization constraint for $\Psi$ gives a Schrödinger equation:

$$
H(\mu) \Psi(\mu)=E(\mu) \Psi(\mu)
$$

The eigenfunction $\Psi(\mu)$ is the solution to the minimization problem eq 1 . The Hamilton operator

$$
H(\mu)=T+V(\mu)+W(\mu)
$$

contains a local one-particle operator,

$$
V(\mu)=V_{n e}+\sum_{i}^{N} v_{h x c}\left(r_{i}, \mu\right)
$$

the local potential $v_{h x c}$ being obtained from a functional derivative,

$$
v_{h x c}(r, \mu)=\delta \overline{\mathcal{E}}_{h x c}[n, \mu] / \delta n(r)
$$

In the following, it will be assumed that $W \rightarrow 0$ for $\mu \rightarrow 0$, and that $W \rightarrow V_{e e}$, the electron-electron interaction, when $\mu \rightarrow \infty$. A correctly constructed approximation $\overline{\mathcal{E}}_{h x c}$ vanishes when $\mu \rightarrow \infty$, and in this limit, $\mathcal{E}_{0}$ becomes the exact ground state energy. Please notice that the model system described by the above Hamiltonian also produces excited states, and that in the limit $\mu \rightarrow \infty$ these become the exact excited states.

For exemplification,

$$
W(\mu)=\sum_{i<j} \operatorname{erf}\left(\mu r_{i j}\right) / r_{i j}
$$

is used in this paper; $r_{i j}$ is the distance between electrons. A local density approximation ( $\mu$-dependent LDA [5-7]) is used for the exchange-correlation functional.

\section{Improving the estimate of the exact energy}

As the potential $V(\mu)$ is known once an approximation was chosen for $\overline{\mathcal{E}}_{h x c}$ (eqs 4,5$)$, we can assume that $E(\mu)$, eq 2 , can be obtained accurately. For example, for $W=$ 0 (in a Kohn-Sham calculation using some approximate exchange-correlation potential) it is simply a sum over occupied orbital eigenvalues. As $E(\mu)$ is, in general, not equal to the exact energy, $E(\infty)$, a correction

$$
\bar{E}(\mu)=E(\infty)-E(\mu)
$$

is needed. The correction given by the density functional approximation,

$$
\begin{aligned}
\overline{\mathcal{E}}(\mu) & =\mathcal{E}_{0}-E(\mu) \\
& =\overline{\mathcal{E}}_{h x c}\left[n_{\Psi(\mu)}, \mu\right]-\int d^{3} r n(r) v_{h x c}(r, \mu)
\end{aligned}
$$

$\overline{\mathcal{E}}(\mu)$ is only an estimate of $\bar{E}(\mu)$ because $\overline{\mathcal{E}}_{h x c}$ used in eq 1 is not exact.
We discuss now how to obtain some information about the correction, $\bar{E}(\mu)$, without using the density functional approximation $\overline{\mathcal{E}}_{h x c}$ for it. We choose to make the correction accurate for large, but finite $\mu$ by using the derivative of $\bar{E}(\mu)$ with respect to $\mu, \bar{E}^{\prime}(\mu)$. We first notice that the Hellmann-Feynman theorem permits a relatively cheap accurate determination of $E^{\prime}(\mu)$ without re-computing $\Psi(\mu)$,

$$
E^{\prime}(\mu)=\left\langle\Psi(\mu)\left|\partial_{\mu} V(\mu)+\partial_{\mu} W(\mu)\right| \Psi(\mu)\right\rangle
$$

Second, we notice that $E(\mu)+\bar{E}(\mu)=E(\infty)$ does not depend on $\mu$, and thus, although $\bar{E}(\mu)$ is not known, accurate information about $\bar{E}^{\prime}(\mu)$ is available through the derivative of $E(\mu)$ with respect to $\mu, E^{\prime}(\mu)$,

$$
E^{\prime}(\mu)+\bar{E}^{\prime}(\mu)=0
$$

We can gain in accuracy if we posses information about the behavior of $\bar{E}(\mu)$. For example, assume that around a given $\mu$, we know how the exact correction behaves,

$$
\bar{E}(\mu)=c f(\mu)+\ldots
$$

where $f$ is a known function, and the constant $c$ is system-dependent. In particular, we not only know that for $W$ of eq 6 , at large $\mu, \bar{E}(\mu)$ vanishes, but also know the decay rate with $\mu$ (see, e.g., $[5,8,9]$ ),

$$
\bar{E}(\mu)=c \mu^{-2}+\mathcal{O}\left(\mu^{-3}\right)
$$

We now use the derivative of $\bar{E}(\mu)$ with respect to $\mu$,

$$
\bar{E}^{\prime}(\mu)=c f^{\prime}(\mu)+\ldots
$$

to extract the coefficient $c=\bar{E}^{\prime}(\mu) / f^{\prime}(\mu)$, and obtain [10]

$$
\bar{E}(\mu)=\frac{f(\mu)}{f^{\prime}(\mu)} \bar{E}^{\prime}(\mu)+\ldots
$$

For our specific example, $W$ of eq 6 , at large $\mu$,

$$
\bar{E}^{\prime}(\mu)=-2 c \mu^{-3}+\mathcal{O}\left(\mu^{-4}\right)
$$

and

$$
\bar{E}(\mu)=-\frac{1}{2} \mu \bar{E}^{\prime}(\mu)+\mathcal{O}\left(\mu^{-3}\right)=\frac{1}{2} \mu E^{\prime}(\mu)+\mathcal{O}\left(\mu^{-3}\right)
$$

where eq 11 has been used for the last equality.

Let us now consider the behavior at large $\mu$ when approximations are used, $\overline{\mathcal{E}}(\mu \rightarrow \infty)$, eq 9 . For our choice of interaction, eq 6 , we know that the correction to the electron-electron interaction term,

$$
\left\langle\Psi(\mu \rightarrow \infty)\left|V_{e e}-W(\mu \rightarrow \infty)\right| \Psi(\mu \rightarrow \infty)\right\rangle
$$

is proportional to

$$
\mu^{-2}\left\langle\Psi(\infty)\left|\sum_{i \neq j} \delta\left(\left|r_{i}-r_{j}\right|\right)\right| \Psi(\infty)\right\rangle
$$


i.e., to $\mu^{-2}$, and to the system average of the on-top pair density of the exact system (see, e.g., $[8,9]$ ). Let us consider $\mu$-dependent approximations for $\overline{\mathcal{E}}_{h x c}$ that model pair-densities (like LDA). In them, the error originates from using the system-average of the model pair density, instead of that of the system under consideration. Thus, $\overline{\mathcal{E}}_{h x c}$ is proportional to $\mu^{-2}$,

$$
\overline{\mathcal{E}}_{h x c} \propto \mu^{-2}+\mathcal{O}\left(\mu^{-3}\right)
$$

Eq 9 also contains $v_{h x c}$, eq 5 . The proportionality to $\mu^{-2}$ remains after taking the functional derivative wrt the density, in order to produce $v_{h x c}$. It follows that $\overline{\mathcal{E}}$ also decays as $\mu^{-2}$, and we can use, for $\overline{\mathcal{E}}(\mu \rightarrow \infty)$, the same expression as for the exact correction, eq 17, viz.,

$$
\overline{\mathcal{E}}(\mu)=-\frac{1}{2} \mu \overline{\mathcal{E}}^{\prime}(\mu)+\mathcal{O}\left(\mu^{-3}\right)
$$

In this expression, too, the derivative has introduced the information about the proportionality constant.

In order to analyze the error of $\overline{\mathcal{E}}_{h x c}$ when $\mu \rightarrow \infty$ we decompose it into a Hartree, an exchange and a correlation part. In this regime, the approximation probes the on-top pair density of the model, cf. eq 18. The Hartree part is evidently given by the density. At coalescence, the reduced first-order density matrix becomes equal to the density, and thus, for a closed shell, the exchange part of the pair density is also determined by the density. For a closed shell, the only error shows up in the correlation part of the pair density $[5,9,11]$. For open shells, however, the exchange component is not determined by the density alone, but by the spin-components of the density. This is why density functional approximations rely not on the density alone, but also on spin-density. It is wellknown, however, that the dependence on spin-density, introduces new problems related to size-consistency (see, e.g., $[6,12,13])$. One thus has the dilemma of either seriously losing numerical accuracy, or size-consistency. In the present paper, spin-density is not used, in order to favor size-consistency. For describing the correlation part of $\overline{\mathcal{E}}_{h x c}$ errors are present. In LDA, it is modeled using the correlation hole of the uniform electron gas. This is a good approximation, but only an approximation as becomes evident, e.g., for one-electron systems.

We can try to introduce some correct information about the system obtained from the energy derivative, as in eq 17, to replace the false one contained in the model system used in the density functional approximation. First, we consider expanding both $\bar{E}$ and $\overline{\mathcal{E}}$ in some (complete) basis,

$$
\begin{aligned}
& \bar{E}(\mu)=\sum_{i} \bar{c}_{i} \chi_{i}(\mu) \\
& \overline{\mathcal{E}}(\mu)=\sum_{i} \overline{\mathfrak{c}}_{i} \chi_{i}(\mu)
\end{aligned}
$$

We now identify the basis function, $\chi_{j}$, that is responsible for the behavior we are interested in (at large $\mu$ ).
As we can consider that we can obtain its coefficient $\bar{c}_{j}$ accurately, we replace the approximate coefficient in the expression of $\overline{\mathcal{E}}$ by an accurate estimate of one of the coefficients, $\bar{c}_{j}$,

$$
\bar{E}(\mu) \approx \overline{\mathcal{E}}(\mu)+\left(\bar{c}_{j}-\overline{\mathfrak{c}}_{j}\right) \chi_{j}(\mu)
$$

To determine the coefficients, we use the derivatives, eq 15 , and write

$$
\bar{E}(\mu) \approx \overline{\mathcal{E}}(\mu)+\frac{\chi_{j}(\mu)}{\chi_{j}^{\prime}(\mu)}\left(\bar{E}^{\prime}(\mu)-\overline{\mathcal{E}}^{\prime}(\mu)\right)
$$

When choosing $W$ as in eq 6 , and making the approximation valid for large $\mu$, by eqs 17 and 20 ,

$$
\bar{E}(\mu) \approx \overline{\mathcal{E}}(\mu)-\frac{1}{2} \mu\left(\bar{E}^{\prime}(\mu)-\overline{\mathcal{E}}^{\prime}(\mu)\right)
$$

The same equation could be produced by requesting that the approximation deviates minimally from the density functional approximation, in a least-squares sense, with the constraint that the derivative of the approximation with respect to $\mu$ corresponds to the exact one.

Please notice that in the present construction, density functional approximations were used to construct model systems. The consistency between the correction to the energy and the potential present in density functional theory is not needed. Thus, the present approach can be applied to ground state, and to excited states, as well.

The derivative is an additional information from which we can infer from $E$ in a given point $\mu$ the behavior in the vicinity of it. This procedure can be continued with higher derivatives, or in a computationally more convenient way, repeated for different $\mu$ points, in order to improve the accuracy in a systematic way. However, in the present paper, only the first correction, using only $E^{\prime}(\mu)$ in a single point will be presented.

\section{NUMERICAL RESULTS}

In this section, the approximation of eq 25 is tested for some two-electron systems. We know that this approximation becomes exact for large $\mu$. The computations explore the behavior outside this regime. Its importance is related to the ansatz made for $W$ : as long as $W$ is weak, the computational effort is expected to be small.

In order not to bias the results, relatively large atomic basis sets were used (cc-pV5Z [14]). Furthermore, the wave function $\Psi(\mu)$ was computed with a full configuration interaction calculation (range-separated configuration interaction program of the Molpro code [15-24]). All the plots show the error done by approximating $\bar{E}(\mu)$ by using eq 25 , instead of $E(\infty)-E(\mu)$, for different values of $\mu$. By construction, the errors vanish at large $\mu$. As the correction is based on an expansion at large $\mu$, one will not expect it to work for $\mu$ smaller than $\approx 1$. As evident from eq 25, no correction to the density functional expression is present at $\mu=0$. 


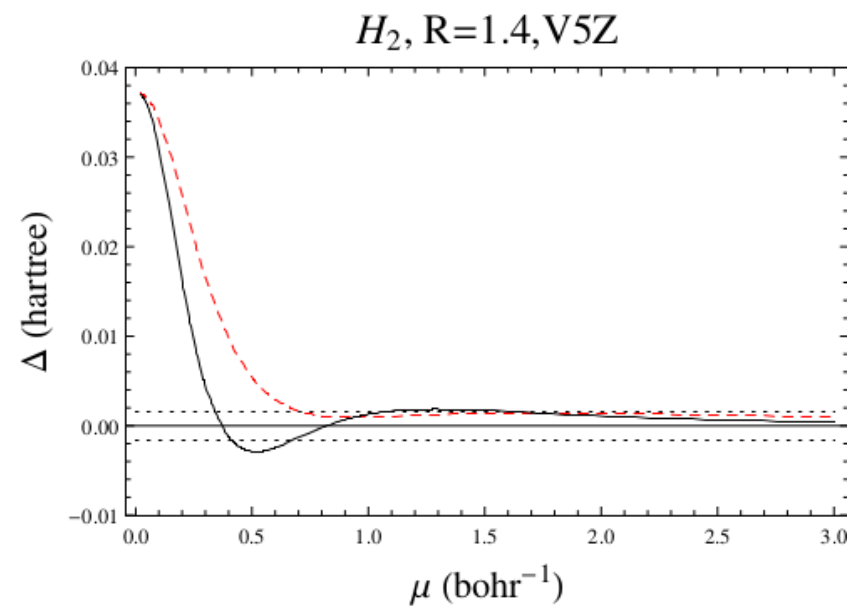

FIG. 1: Error of the approximation, eq 25, as a function of the parameter of the model Hamiltonian $(\mu)$ for the hydrogen molecule, in its ground state, at the equilibrium distance (full curve), and error of the LDA functional (dashed curve). The horizontal dotted lines show the limits of "chemical accuracy" $( \pm 1 \mathrm{kcal} / \mathrm{mol})$.

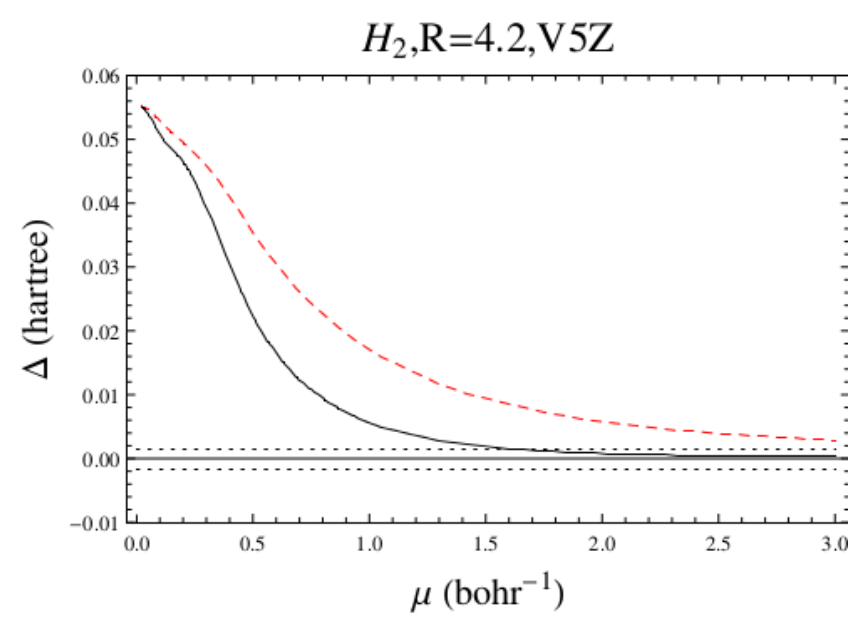

FIG. 2: Same as fig 1, but the bond is now stretched to three times the equilibrium distance.

The first example is that of the hydrogen molecule at the equilibrium distance (fig 1 ). It shows exactly the features expected. We can see in fig 1 that the approximation has a quality comparable to that short-range LDA, known to work well for larger values of $\mu$.

However, when the hydrogen molecule is stretched, the broken symmetry solution and spin-density has to be introduced in LDA to describe the potential energy curve at LDA level. We see in fig 2 that keeping a correct (zero) spin-density, the LDA error is relatively large when the distance between the hydrogen nuclei increases. In this case, the approximation of eq 25 provides improved results. In the limit of dissociation, the error would be twice that obtained for hydrogen atoms, as sizeconsistency is respected.

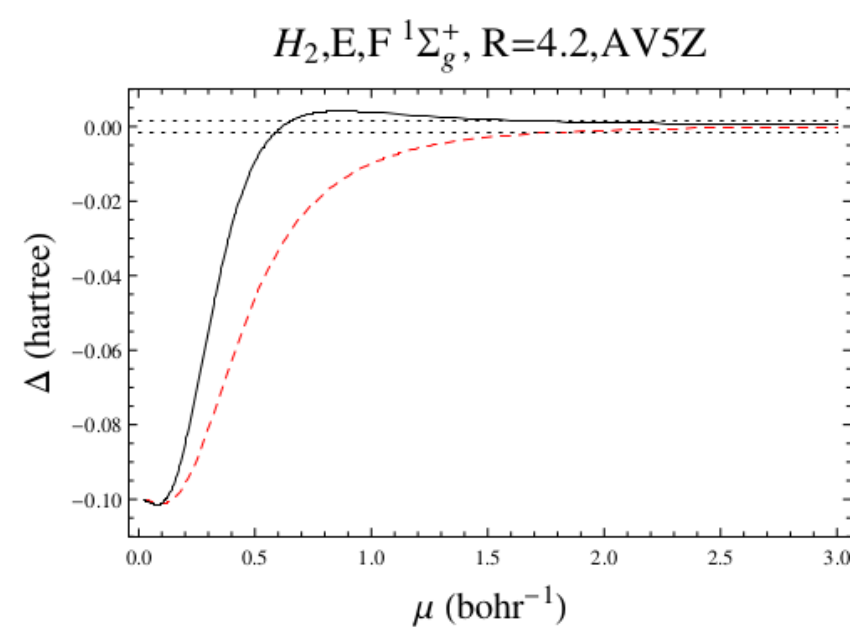

FIG. 3: Same as fig 1, but the hydrogen molecule is now in the $E, F^{1} \Sigma_{g}^{+}$excited state, around the outer minimum of the potential energy curve, 4.2 bohr [25].

The next example treats an excited state of the hydrogen molecule, $E, F^{1} \Sigma_{g}^{+}$. It corresponds to the resonance structure $\mathrm{H}^{+} \ldots \mathrm{H}^{-} \leftrightarrow \mathrm{H}^{-} \ldots \mathrm{H}^{+}$. In this case, LDA is not expected to work, because electrons are brought together by correlation, while a transfer of the pair function from the ground state of the uniform electron gas keeps the electrons apart (see fig 3). Furthermore, symmetry breaking cannot help in its standard implementation. However, when using eq 25 the quality is comparable to that seen previously, as long as $\mu$ is not too small.

Finally, let us discuss a problem, known to show up for the forms of $W(\mu)$, where $\mu$ appears as multiplied by the distance between electrons, $\mu r_{12}$ [26]. In such an ansatz one expects a scaling of the error: the error observed for a given $\mu$ for the more diffuse system show up at a larger $\mu$ for a compact system (because, on average, $r_{12}$ is reduced). This can be illustrated by the behavior of the approximation for the He atom (that can be seen as the hydrogen molecule at zero internuclear separation), fig 4 . One can see that the shape of the curve in fig 4 is similar to that of the hydrogen molecule at the equilibrium distance. The point where the large- $\mu$ approximation becomes critical is marked by oscillations. For He, they show up at a larger value of $\mu$ than for $\mathrm{H}_{2}$, and and they are more important (also for $\mu=0$, the Kohn-Sham system, the LDA errors are larger). The curve for the hydrogen molecule and that for the helium atom can be made to be almost superimposed by using a scaling factor of $\approx 2$ for one of them.

As for the hydrogen molecule, the method does not bring any correction over the density functional approximation at $\mu=0$. However, one may notice an improvement over the LDA for small values of $\mu$, also for He. 


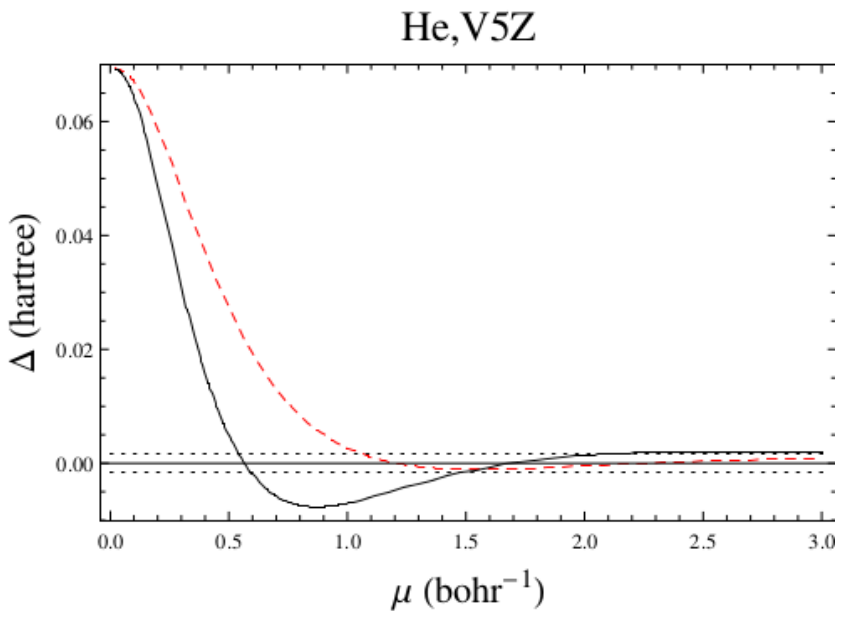

FIG. 4: Same as fig 1, but now for the helium atom.

\section{SUMMARY AND COMPARISONS}

It is proposed to consider density functionals as models for producing model energies, $E(\mu)$, eq 2. To obtain $E(\mu)$, the potential produced by the density functional is used, not the functional itself. To obtain the energy of the physical system, a correction is needed. It is proposed to correct the approximate density functional expression by considering changes in the model obtained by changing $\mu$, introducing this way system-dependent information (see the constant $c$ in eq 12). In particular, one can use the derivative of the model energy with respect to $\mu$, as in eq 15 . The same procedure can be applied also for approximations, and the the wrong behavior of the approximation (in the regime considered) can be eliminated (eq 24, or eq 25). It is a first step in correcting approximate density functionals, by introducing more information from model systems.

The approximation introduced in this paper does not require the use of spin-density, and is not restricted to ground states.

In order to produce numerical examples, a Hamiltonian containing long-range interaction $\left(\operatorname{erf}\left(\mu r_{12}\right) / r_{12}\right)$ was used. The correction introduced becomes exact as this interaction approaches the physical one, $1 / r_{12}$.

In the regime where the approximation becomes exact, one could as well use eq 17 (as in [10]). The advantage of eq 25 is that, in contrast to eq 17 , a correction is active even for small $\mu$. However, for the important limit $\mu=$ 0 , there is no improvement over the density functional approximation.

Of course, this approach can be extended to density functionals that work better than LDA. One can thus expect improving the quality of the approximation when the interaction is weak.

Finally, it should be mentioned that the form of $H(\mu)$ used in this paper is not the only one of interest. For example one could consider model Hamiltonians of the form $T(\mu)+V(\mu)+V_{e e}$ where $T(\mu)$ is a non-local oneparticle operator. It has be shown that it can lead to good approximations [27-31].

\section{ACKNOWLEDGMENT}

J. Toulouse (UPMC Univ Paris 06, France) and the two anonymous referees have contributed by their questions and suggestions to improve the clarity of this paper.
[1] P. Hohenberg and W. Kohn, Phys. Rev. 136, B 864 (1964).

[2] S.-K. Ma and K. A. Brueckner, Phys. Rev. 165, 165 (1968).

[3] D. C. Langreth and M. J. Mehl, Phys. Rev. B 28, 1809 (1983).

[4] A. D. Becke, The Journal of Chemical Physics 98, 1372 (1993).

[5] P. M. W. Gill, R. D. Adamson, and J. A. Pople, Mol. Phys. 88, 1005 (1996).

[6] A. Savin, in Recent Developments of Modern Density Functional Theory, edited by J. M. Seminario (Elsevier, Amsterdam, 1996), pp. 327-357.

[7] S. Paziani, S. Moroni, P. Gori-Giorgi, and G. B. Bachelet, Phys. Rev. B 73, 155111 (2006).

[8] P. Gori-Giorgi and A. Savin, Phys. Rev. A 73, 032506 (2006).

[9] J. Toulouse, F. Colonna, and A. Savin, Phys. Rev. A 70, 062505 (2004).

[10] A. Savin, J. Chem. Phys. 134, 214108 (2011).

[11] R. Pollet, F. Colonna, T. Leininger, H. Stoll, H.-J. Werner, and A. Savin, Int. J. Quantum. Chem. 91, 84
(2003).

[12] J. P. Perdew, in Density Functional Methods in Physics. Proceedings of a NATO ASI held 1983 in Alcabideche, Portugal, edited by R. Dreizler and J. da Providencia (Plenum Press, New York, 1985), p. 265-308.

[13] A. J. Cohen, P. Mori-Sánchez, and W. Yang, Science 321, 792 (2008).

[14] T. H. Dunning, J. Chem. Phys. 90, 1007 (1989).

[15] H.-J. Werner, P. Knowles, R. Lindh, F. R. Manby, M. Schütz, P. Celani, T. Korona, A. Mitrushenkov, G. Rauhut, T. Adler, et al., Molpro, version 2009.1 a package of ab initio programs (2009), see http://www.molpro.net.

[16] M. Schütz, R. Lindh, and H.-J. Werner, Mol. Phys. 96, 719 (1999).

[17] H.-J. Werner and P. Knowles, J. Chem. Phys. 89, 5803 (1988).

[18] P. J. Knowles and H.-J. Werner, Chem. Phys. Lett. 514, 145 (1988).

[19] H.-J. Werner and E.-A. Reinsch, J. Chem. Phys. 76, 3144 (1982).

[20] H.-J.Werner, Adv. Chem. Phys. LXIX, 1 (1987). 
[21] R. Polly, H.-J. Werner, F. Manby, and P. Knowles, Mol. Phys. 102, 2311 (2004).

[22] T. Leininger, H. Stoll, H.-J. Werner, and A. Savin, Chem. Phys. Lett. 275, 151 (1997).

[23] J. G. Ángyán, I. C. Gerber, A. Savin, and J. Toulouse, Phys. Rev. A 72, 012510 (2005).

[24] E. Goll, H.-J. Werner, and H. Stoll, Phys. Chem. Chem. Phys. 7, 3917 (2005).

[25] E. R. Davidson, J. Chem. Phys. 33, 1577 (1960).

[26] A. Savin and H.-J. Flad, Int. J. Quantum. Chem. 56, 327 (1995).

[27] J. Rey and A. Savin, Int. J. Quantum. Chem. 69, 581
(1998).

[28] J.B. Krieger, J. Chen, G. Iafrate, and A. Savin, in Electron Correlation and Material Properties, edited by A. Gonis, and N. Kioussis (Plenum, New York, 1999), p. 463.

[29] P. Y. Ayala, G. E. Scuseria, and A. Savin, Chem. Phys. Letters 307, 227 (1999).

[30] S. S. Iyengar, G. E. Scuseria, and A. Savin, International Journal of Quantum Chemistry 79, 222 (2000).

[31] C. Gutlé and A. Savin, Phys. Rev. A 75, 032519 (2007). 